\title{
Vitamin $D$ as an Adjunctive Treatment to Standard Drugs in Pulmonary Tuberculosis Patients: An Evidence-Based Case Report
}

\author{
Diajeng Ayesha Soeharto, ${ }^{1}$ Diana Ashilah Rifai, ${ }^{1}$ Stella Marsudidjadja, ${ }^{1}$ \\ Aisha Emilirosy Roekman, ${ }^{1}$ Chadijah Karima Assegaf, ${ }^{1}$ and Melva Louisa ${ }^{2}{ }^{2}$ \\ ${ }^{1}$ Medical Student, Faculty of Medicine, Universitas Indonesia, Indonesia \\ ${ }^{2}$ Department of Pharmacology and Therapeutics, Faculty of Medicine, Universitas Indonesia, Indonesia
}

Correspondence should be addressed to Melva Louisa; melva.louisa@gmail.com

Received 30 November 2018; Revised 19 March 2019; Accepted 21 May 2019; Published 20 June 2019

Academic Editor: Ultan Power

Copyright (c) 2019 Diajeng Ayesha Soeharto et al. This is an open access article distributed under the Creative Commons Attribution License, which permits unrestricted use, distribution, and reproduction in any medium, provided the original work is properly cited.

Background. Vitamin D has a prominent role in the body's innate immunity as it is important in the maintenance of macrophages and monocytes and its function in defending against infections. In-vitro studies have established vitamin D's potential role in tuberculosis (TB) infection, in that it restricts Mycobacterium tuberculosis growth, thus implying the potential benefit of vitamin D as an adjunctive treatment for TB. However, clinical trials and reviews have contradicting findings regarding the true clinical efficacy of adjunctive vitamin D, particularly in reducing the sputum conversion rate (SCR). Objective. This study aims to update the current evidence regarding vitamin $\mathrm{D}$ supplementation as an adjunctive treatment in achieving the smear sputum conversion rate (SCR) among pulmonary TB patients. Method. A comprehensive search was conducted in October 2018 in PubMed-NCBI, MEDLINEOVID, SCOPUS-Elsevier, and Cochrane. The selection of studies was done as per the predetermined inclusion and exclusion criteria of this EBCR and resulted in the inclusion of 11 eligible studies (8 RCTs and 3 systematic reviews). The selected studies were then critically appraised for their validity, importance, and applicability according to the CEBM (Centre for Evidence-Based Medicine) appraisal tools. Results. Overall, most of the trials showed no statistically significant changes in terms of the proportion of TB patients with a negative sputum smear conversion in the group treated with an adjunctive therapy vs. the group treated with standard antituberculosis therapy alone. Only one trial showed significant results, which was conducted in a population of TB patients with vitamin D deficiency. Furthermore, overall the reviews showed no significant change in the 8-week sputum smear conversion after treatment within the group given vitamin $\mathrm{D}$ in comparison to those who were not. Conclusion. Vitamin $\mathrm{D}$ as adjunctive therapy in TB patients shows no clinical improvement in terms of sputum conversion to antituberculosis management.

\section{Introduction}

A woman came to the clinic with her 41-year old husband. Her husband was diagnosed with pulmonary tuberculosis (TB) last month and had come back for a follow-up consult. The husband reported that he had been routinely taking the prescribed anti-TB drugs since then. During the consultation, she mentioned that she read some information online regarding the benefits of vitamin D supplements for TB patients. She was wondering whether or not vitamin $\mathrm{D}$ supplements could improve her husband's treatment outcome if he were to take them along with his anti-TB drugs.

\section{Background}

Tuberculosis infection (TB) is one of the top ten causes of death worldwide and is due to Mycobacterium tuberculosis (Mtb) infection. Although this bacterium can affect numerous organs, the respiratory system remains the most prominently affected. TB is a contagious disease, and air serves as the main mode of transmission [1].

Due to its ease of transmission, early prevention and treatment are crucial. Early diagnosis can be done since a susceptible patient is suspected of having a TB infection. The examination includes taking a history, physical examination, 
radiological chest examination, and the laboratory culture of Mycobacterium tuberculosis by culture of sputum or tissue biopsy. A sputum smear test can also be conducted to assess the degree of infection. Treatment for tuberculosis includes a combination of drugs administered for at least 6 months, and the conversion of sputum smear test (SSC) from positive to negative indicates successful treatment and generally occurs after two months of treatment $[2,3]$. The two-month sputum conversion rate is currently considered as the preferred endpoint because this marker has a significant correlation with combined response rates, relapse, and intermediate bacteriological endpoint [2]. Standard treatment for TB consists of 4 antituberculosis drugs, which are isoniazid, rifampin, pyrazinamide, and either ethambutol or streptomycin. This regimen is given for 2 months with 60 doses, followed by 4 months of treatment with only isoniazid and rifampin with 120 doses. The choice of treatment depends on the patients' drug susceptibility and the response following treatment [1, 4].

Vitamin D has a vital role in the body's natural defense mechanism towards infection since it promotes the role of macrophages and monocytes which is important in pathogenesis [5]. In infection caused by Mycobacterium tuberculosis (Mtb), calcidiol, the major circulating metabolite of vitamin $\mathrm{D}$, supports the induction of innate antimicrobial immune response by causing $\mathrm{Mtb}$ growth restriction. Calcidiol is metabolized by CYP27B1 into its active form, calcitriol [6]. Calcitriol exerts its antimicrobial activity by modulating the reactive oxygen intermediates, the induction of antimicrobial peptides, and autophagy [7]. Calcitriol plays a key role in the maintenance of immune homeostasis and in tuberculosis and works indirectly by suppressing MHC class II and directly suppresses IFN- $\gamma$ and IL-2 from CD4 Th1 cells [8]. Coussens et al. have also shown that high dose of vitamin D supplementation suppresses antigen-stimulated proinflammatory cytokine responses and accelerates the resolution of IL-4, CC chemokine ligand 5, and IFN- $\alpha$ secretion during the administration of antituberculosis drugs [9].

Thus, some studies have suggested adjunct vitamin D supplementation to enhance the response to antituberculosis therapy [10]. However, several studies have differing results regarding the impact of vitamin $\mathrm{D}$ as adjunctive therapy to $\mathrm{TB}$ patients [11]. Therefore, the purpose of this article is to update and clarify the impact of adjunct vitamin D supplementation in achieving an early smear sputum conversion rate among pulmonary TB patients.

\section{Method}

3.1. Search Strategy. A comprehensive literature search was conducted in October 2018 on four electronic databases: PubMed-NCBI, MEDLINE-OVID, SCOPUS-Elsevier, and Cochrane by using keywords and Mesh terms as follows: “Tuberculosis," "Vitamin D," (Sputum OR "Sputum Conversion Rate" OR SCR). The keywords were then incorporated with Boolean operators into ("Tuberculosis"[MeSH]) AND "Vitamin D"[MeSH] AND (Sputum OR "Sputum Conversion Rate" OR SCR), or an equivalent of that to suit each database.
A search limitation was not applied in this search strategy to avoid overlooking any articles. The search results', from all four electronic databases, all duplicated articles were first removed, and the results were then screened by evaluating the title and abstract with regard to the eligibility criteria of this EBCR (outlined in Figure 1). The final inclusion of studies was determined by examining each full article and evaluating whether or not the study satisfied the inclusion criteria.

3.2. Eligibility Criteria. The targeted population was patients with a Tuberculosis infection who were undergoing antiTB therapy with the sputum smear conversion rate as the outcome measure. The inclusion criteria for the research evidence were randomized controlled trials (RCT), systematic reviews, and meta-analysis studies which compare two different interventions: standard combination anti-TB drug therapy versus standard combination anti-TB drug therapy with vitamin $\mathrm{D}$ supplementation. The participants of the study were expected to have the absence of any predisposing illness (such as Type II Diabetes Mellitus). Moreover, the study with an adjuvant therapy to combine with vitamin D supplementation for the participants in the intervention group was considerably biased and thus excluded. Articles were excluded if the papers included patients with comorbidities or secondary chronic infections such as HIV, HBV, or $\mathrm{HCV}$, they were animal or in-vitro studies, the full-text was not available, and if the study did not incorporate a sputum smear conversion as the end-point measure.

3.3. Critical Appraisal. The CEBM (Centre for EvidenceBased Medicine) appraisal tools [22] were used to systematically evaluate the quality of evidence included in this EBCR. CEBM appraisal sheets for RCTs and systematic review sheets were used with respect to each study type. As for the RCT critical appraisal sheet, the quality was evaluated within the domains of internal validity (mainly focusing on randomization, blinding, and comparability) and clinical importance (study findings). Furthermore, systematic reviews were evaluated with regard to the domains of a comprehensive and appropriate search strategy, validity, and heterogeneity of included studies, as well as the conclusion drawn by the review.

\section{Results}

4.1. Search Findings. As shown in Figure 1, the initial search of four electronic databases using the aforementioned search terms yielded 204 potentially relevant studies (Table 1) from which 90 duplicate studies were removed. The remaining 112 studies were screened based on the title and abstract, where 97 were excluded as they either did not study adjunctive vitamin $\mathrm{D}$ therapy in TB patients, included patients with other comorbidities, did not evaluate SCR, or were not RCTs, systematic reviews, or meta-analyses. The remaining 17 potentially relevant studies were assessed for eligibility by examining the full-text articles, with regard to the EBCR eligibility criteria. From this, a total of 6 studies were excluded: one study was not a systematic review, one did not have the full-text available, two did not include the SCR as its outcome 


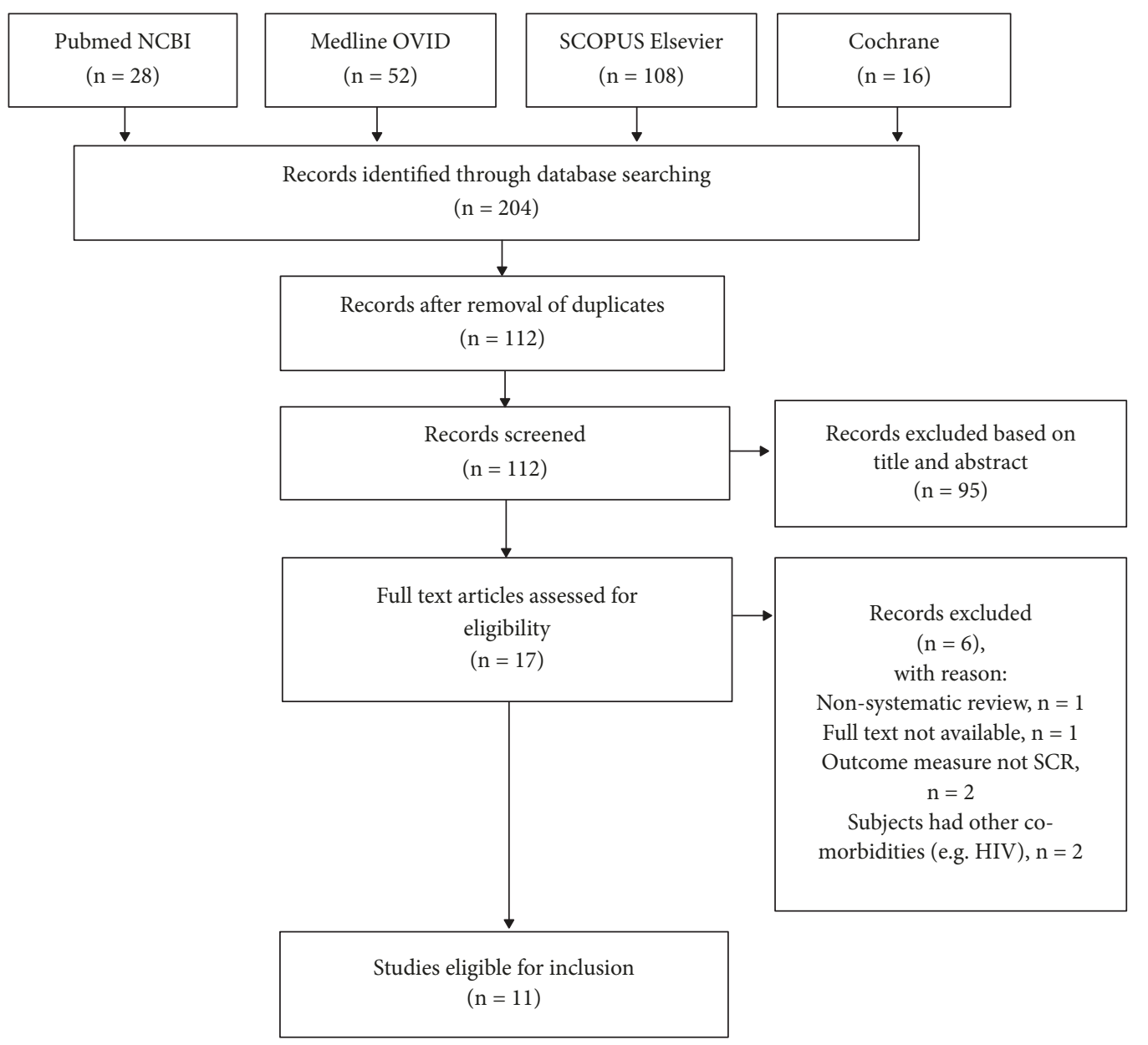

FIGURE 1: PRISMA flowchart outlining the process of article selection.

measure, and two included subjects with other comorbidities. Thus, a total of 11 studies, consisting of three systematic reviews and eight RCTs, were selected and considered eligible for inclusion in this EBCR.

4.2. Critical Appraisal. Out of the eight RCT studies, 6 completely fulfilled the internal validity criteria in the CEBM RCT appraisal tool by the University of Oxford [22] (Table 2). Primarily, the studies (7 out of 8 ) found that there was no significant difference between adjunctive vitamin D3 and the control treatment in achieving a negative sputum culture result by the end of the fixed time ( 2 months), as portrayed by the $95 \%$ CI of the RCTs which crossed the line of no effect, except for the study by Afzal et al. [12].

The number needed to treat (NNT) varied considerably (as shown in Table 2), with 10 and 660 as the minimum and maximum value, respectively, and a median of 43 patients as needed to treat in order for one good outcome of sputum conversion. Notably, the NNT varies greatly from study to study, and although this may be suggestive of the varied quality and design of one study to the other, this range of NNT is also typical of trials involving vitamins and preventive therapy in general; thus the values in our appraised studies are as expected.
Overall, the three included reviews were of varying quality (Table 3 ). While all three reviews used appropriate methods in searching for and including evidence, Wallis et al. [20] failed to assess the quality of the included studies and thus were unable to fulfill the criteria for the included study validity. Furthermore, since Wallis et al.s study was merely a systematic review and did not include a pooled quantitative analysis of the studies, the review failed to fulfill the criteria for the resulting similarity. Wang et al. [21] and Wu et al. [10] were both meta-analyses of relatively good quality. The two differed in that Wang et al. showed that the included studies had no signs of heterogeneity, while Wu et al. showed considerably high levels of heterogeneity; however, it is worth noting that in the Wang et al. pooled quantitative analysis regarding vitamin $\mathrm{D}$ and SCR conversion rates, they did not analyze all five of the included studies in the review and also failed to provide a justification as to why some studies were excluded in the quantitative analysis [21].

4.3. Vitamin D Mode of Administration and Dose. Across all the trials, vitamin $\mathrm{D}$ was given concurrently with typical ATT, and the comparison groups were given ATT alone or along with the control. Vitamin D administered orally in 7 studies, where Ganmaa et al. [14] gave four $3.5 \mathrm{mg}$ tablets 
TABLE 1: Search strategy applied in searching for evidence in the four electronic databases.

\begin{tabular}{|c|c|c|c|}
\hline Database & Search Strategy & Hits & Selected Articles \\
\hline PubMed NCBI & $\begin{array}{l}\text { ("Tuberculosis"[Mesh]) AND “Vitamin D”[Mesh] AND } \\
\text { (Sputum OR "Sputum Conversion Rate" OR SCR) }\end{array}$ & 28 & 7 \\
\hline Medline OVID & $\begin{array}{l}\text { 1. TUBERCULOSIS/or Tuberculosis.mp. } \\
\text { 2. Vitamin D/or Vitamin D.mp. } \\
\text { 3. (“Sputum Conversion Rate" or Sputum* or SCR).mp. } \\
\text { [mp=title, abstract, original title, name of substance word, } \\
\text { subject heading word, floating sub-heading word, keyword } \\
\text { heading word, protocol supplementary concept word, rare } \\
\text { disease supplementary concept word, unique identifier, } \\
\text { synonyms] } \\
\text { 4.1 AND 2 AND } 3\end{array}$ & 52 & 3 \\
\hline $\begin{array}{l}\text { SCOPUS } \\
\text { Elsevier }\end{array}$ & $\begin{array}{l}\text { TITLE-ABS-KEY (Tuberculosis AND “Vitamin D” AND } \\
\text { (Sputum OR “Sputum Conversion Rate" OR SCR)) }\end{array}$ & 108 & 1 \\
\hline Cochrane & $\begin{array}{l}\text { (MeSH descriptor: [Tuberculosis]: explode all trees) AND } \\
\text { (MeSH descriptor: [Vitamin D]: explode all trees) AND } \\
\text { Sputum }\end{array}$ & 16 & 0 \\
\hline
\end{tabular}

with a total dose of 140,000 IU of vitamin D3, Nursyam et al. [17] gave oral tablets of $0.25 \mathrm{mg}$ per day for six weeks, Ralph et al. [18] gave one tablet every four weeks of "Calciferol Strong ${ }^{\circledR}$ " (50,000 IU, $\left.1250 \mathrm{mcg}\right)$, and Tukvadze et al. [19] gave $1.25 \mathrm{mg}(50,000 \mathrm{IU})$ of vitamin D3. Other oral modes of administration included the use of $2.5 \mathrm{mg}$ of vitamin $\mathrm{D}$ oil (10,000 IU) given every fortnight for 6 weeks in the study by Daley et al. [13], Martineau et al. [15] used oral vigantol oil, which is similar to that of Mily et al. [16] who used the same type of oil, but at 5,000 IU given once daily. Vitamin D was given intramuscularly in one study by Afzal et al. [12], in which four separate large doses of 10,000 IU were given every fortnight (Table 4). As for the included reviews, the study populations were treated similarly in terms of vitamin $D$ administration to that of the aforementioned trials. All three reviews included both oral and intramuscular administration of vitamin $\mathrm{D}$ and had a range of doses that differed in each study; they were typically given during the intensive phase of TB treatment ( 8 weeks) and administered every one to two weeks (see Table 5).

4.4. Vitamin D and Sputum Conversion Rate. The included RCTs, as shown in Table 4, show that the outcome measure of all the studies was similar and monitored the sputum conversion from positive to negative at different times. For instance, Ralph et al. [18] measured this conversion at the 4 th week and followed up at the 8th week after intervention, whereas Tukvadze et al. [19] and Ganmaa et al. [14] identified the changes at the 8th week. In addition, three other studies by Nursyam [17], Mily et al. [16], and Afzal et al. [12] did repeated examinations of the sputum conversion. Although the treatment follow-ups were done at different frequencies and timepoints, all the studies have SCR endpoints at 8 weeks. Thus, the calculation of NNT and the 95\% CI are based on SCR at the 8th week after treatment.

Almost all the results of these RCTs showed no significant impact of delivering vitamin $\mathrm{D}$ as adjunctive therapy along with ATT to the early sputum conversion rate compared with the standalone consumption of ATT. Conversely, one study by Afzal et al. [12] did show a small benefit of vitamin $\mathrm{D}$ consumption compared to the control group to those receiving vitamin $\mathrm{D}(11.7 \%$ vs. $1.7 \%, \mathrm{p}=0.028)$. Albeit, this study concerned only vitamin D deficient patients. Moreover, Nursyam et al. [17] also showed significant differences in the proportion of negative SCR observed between vitamin D and the placebo group $(100 \%$ vs. $76.7 \%, \mathrm{p}=0.002)$ only at the 6 th week after treatment; however at the 8th week, only 1 patient in the placebo group did not have an SCR (100\% vs. $96.9 \%$; p $=0.99)$. The reviews, on the other hand, had contradicting results (see Table 5). While the review by Wang et al. was suggestive of a significantly higher proportion of sputum conversion in those treated with vitamin $\mathrm{D}(\mathrm{OR}=1.21,95 \%$ $\mathrm{CI}=1.05-1.39, \mathrm{p}=0.007)$, this finding is not in line with the results of the other two newer reviews. The qualitative analysis by Wallis et al. analysis concluded that vitamin $\mathrm{D}$ supplements were not of benefit in terms of the earlier sputum conversion, and this is further corroborated with the findings of Wu et al's pooled qualitative analysis that showed that vitamin D supplementation had no influence on the improvement of sputum smear-negative conversion rates ( $R R$ $=0.99 ; 95 \% \mathrm{CI}=0.91$ to $1.07 ; p=0.77)$. Additionally, Wallis et al. also mentioned potentially concerning incidences of the side-effects along with vitamin D supplements, although they were regarded as safe overall.

\section{Discussion}

Across all of the appraised RCTs, only the study by Afzal et al. [12] had significant clinical importance as indicated by the confidence interval and relative risk (as shown in Table 2). We thought that the main reason that Afzal et al. showed significant effects was that the study population is vitamin $\mathrm{D}$ deficient, while other studies administered vitamin $\mathrm{D}$ despite their vitamin $\mathrm{D}$ status. We thought that the mode of administration of vitamin $\mathrm{D}$ might play a role in the difference in results, particularly since Afzal et al. used an intramuscular injection to administer vitamin D. The pharmacokinetic characteristics can potentially highly 


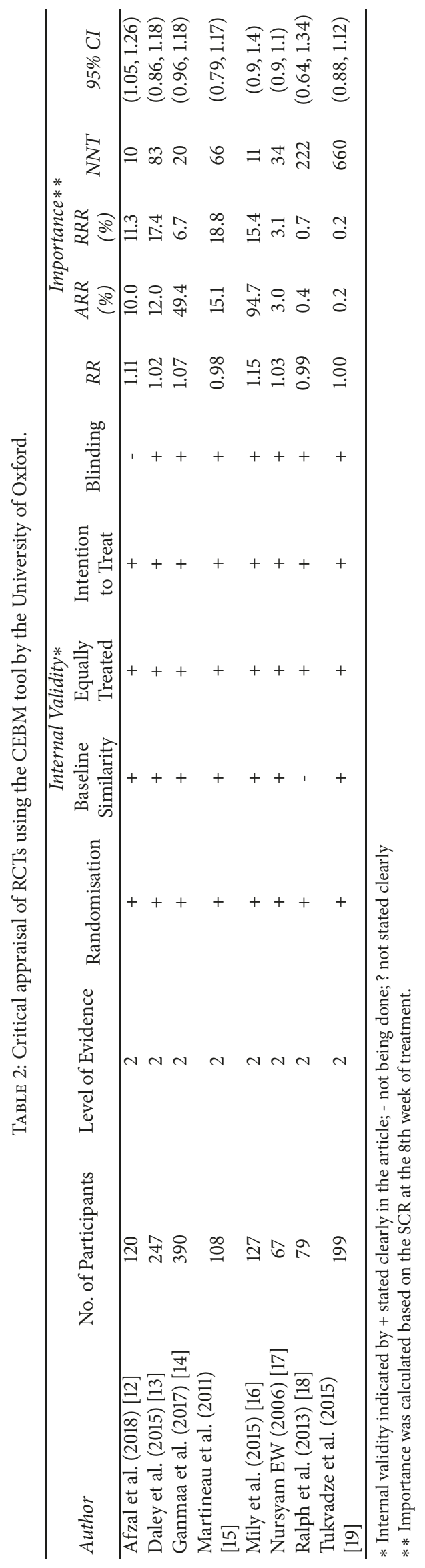









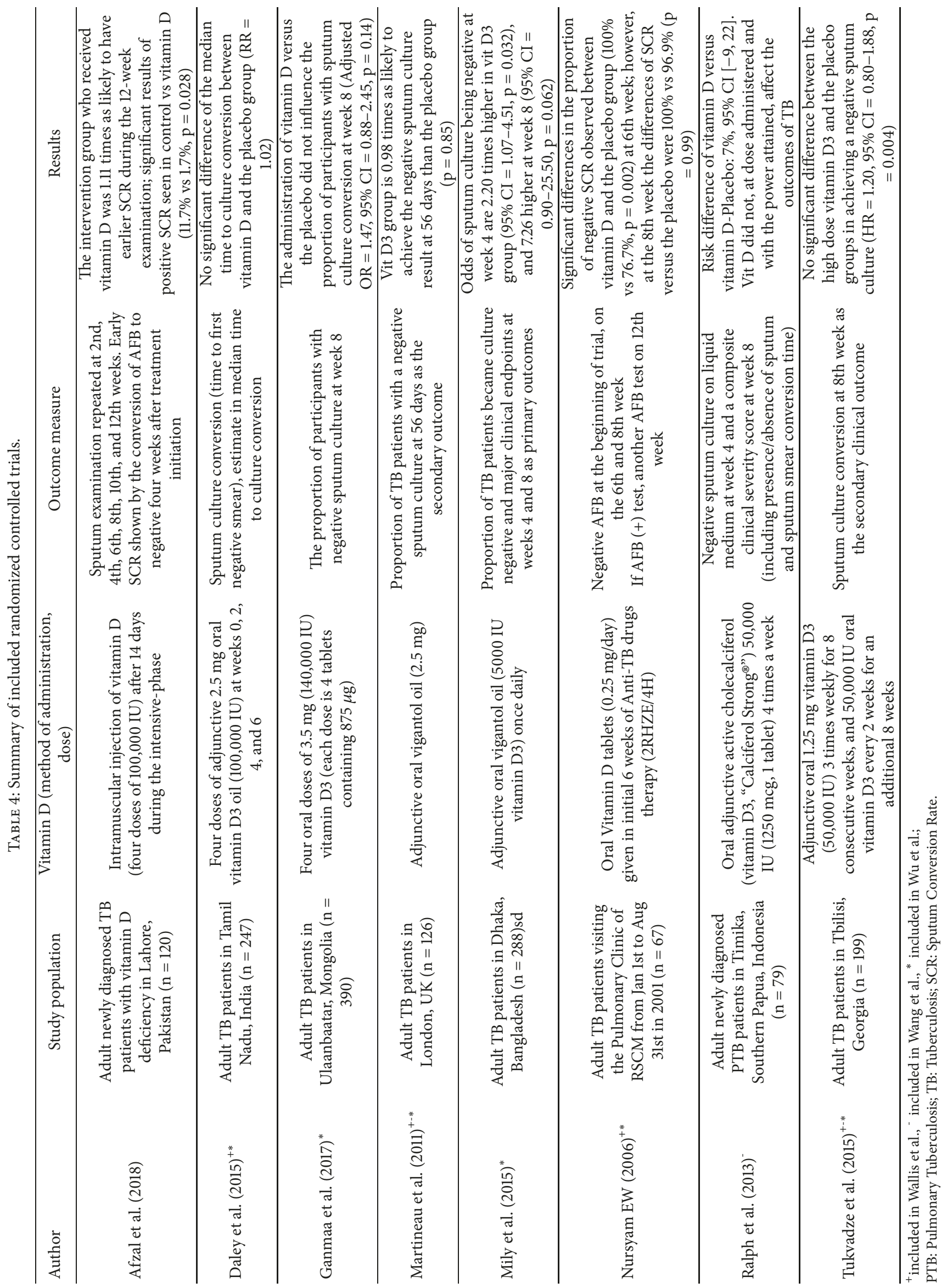




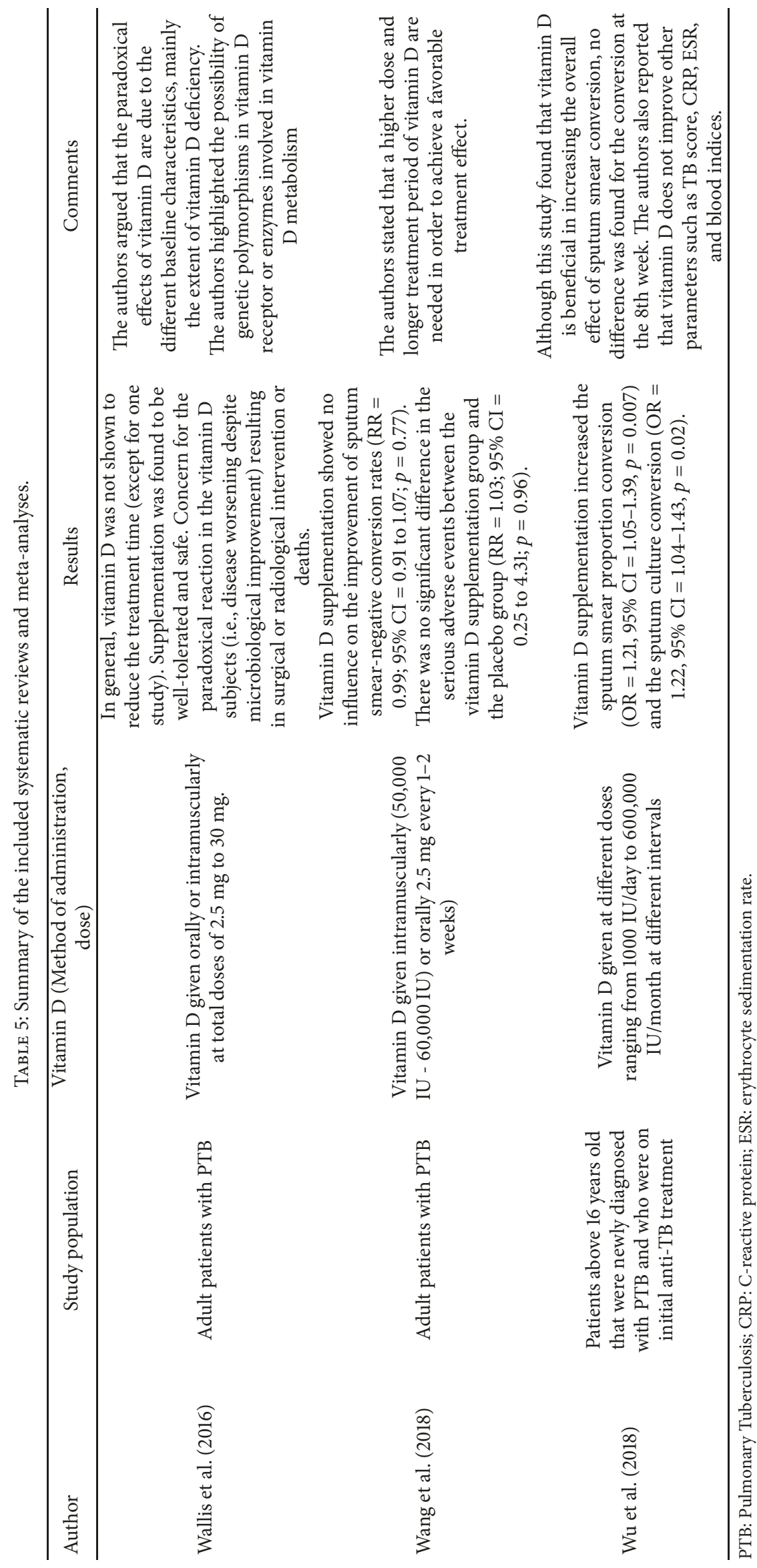


influence sputum conversion results and thus would differ in patients given an intramuscular injection compared to those given oral supplementation. Gupta et al. studied the pharmacokinetic difference of oral versus intramuscular vitamin $\mathrm{D}$ in healthy adults with vitamin $\mathrm{D}$ deficiency and showed that both modes of administration were effective in correcting hypovitaminosis D. However, Gupta et al. showed that intramuscular overtime (after 6 th to 12 weeks of treatment) serum 25-hydroxyvitamin $\mathrm{D}$ was better maintained by intramuscular injection rather than oral administration [23]. Moreover, since the population in the study by Afzal et al. focused only on correcting vitamin $\mathrm{D}$ deficiency rather than vitamin D supplements in the general population, inferences of the study results into the general population cannot be done. Further investigation into the use of vitamin $\mathrm{D}$ intramuscular therapy in the form of RCTs and metaanalyses needs to be conducted in order to establish its true clinical benefit. With the current state of evidence, it would be inefficient to add adjuvant therapy if drastic benefits cannot be reaped from it. Use of IM vitamin D adjuvant therapy is not applicable in some countries; taking into consideration its questionable benefit, the added cost, invasiveness of injections, and especially the added burden of treatment for TB patients (considering that TB is a disease that deals majorly with issues of noncompliance due to the need for multiple drugs over an extended period of time), it cannot be recommended.

Patient selection criteria, dose, mode of vitamin D administration, and methodological differences between the studies might also contribute to the differences in results, which might attenuate the true clinical effect of vitamin D. Most of the studies were done using a small number of subjects, so they were considered as underpowered. The largest study was done by Ganmaa et al. in 390 Mongolian people. The study by Ganmaa et al. confirmed that the genetic variation in vitamin $\mathrm{D}$ metabolizing enzymes CYP27B1 modified the protective effect of vitamin D [14].

Different sputum sampling methods of SCR sampling might also contribute to the difference in the results. The smear samples used were variables between trials. Sputum microscopy is the most common method used to determine SCR $[14,18,19]$, while Daley et al. [13] and Martineau et al. [15] used fluorescent microscopy. The fluorescent microscopy method is higher in sensitivity and faster than conventional microscopy, while sputum microscopy is time-consuming and has a lower sensitivity in HIV coinfection [3].

In interpreting the systematic review by Wallis et al. [20], it is important to take into consideration the differences in the eligibility criteria of the included studies. Although the stated inclusion criteria were comparable to that of this EBCR (where subjects with comorbidities were to be excluded), the review by Wallis et al. had included one study that we had excluded during our selection process due to the inclusion of patients with HIV infection. The immunosuppressive condition of participants might act as a significant confounding factor to the sputum conversion differences in adjunctive vitamin D therapy. Furthermore, another point worth noting is the use of an odds ratio instead of relative risk in the review by Wallis et al. and $\mathrm{Wu}$ et al. which was not justified by the respective authors. Considering that the population and the incidence of the outcomes of the studies were not controlled, the use of an odds ratio would tend to show an overestimation of the true odds and thus would not provide an accurate estimate of the odds.

\section{Conclusion}

Adjunctive vitamin D therapy shows no clinical improvement (sputum conversion) in antituberculosis management. It is not necessary to add oral vitamin $\mathrm{D}$ to the current antituberculosis regimen that the patient takes. Further studies regarding vitamin $\mathrm{D}$ adjunctive therapy in nondeficient TB patients are needed in order to establish the true effect of vitamin $\mathrm{D}$ in the general population of TB patients, particularly evaluating its safety and efficacy.

\section{Conflicts of Interest}

The authors declare that they have no conflicts of interest.

\section{Acknowledgments}

The authors would like to thank Enago (www.enago.com) for the English language review.

\section{References}

[1] Global Tuberculosis Report, World Health Organization, Geneva, Switzerland, Licence: CC BY-NC-SA 3.0 IGO.

[2] N. Rockwood, E. du Bruyn, T. Morris, and R. J. Wilkinson, "Assessment of treatment response in tuberculosis," Expert Review of Respiratory Medicine, vol. 10, no. 6, pp. 643-654, 2016.

[3] P. Cudahy and S. V. Shenoi, "Diagnostics for pulmonary tuberculosis," Postgraduate Medical Journal, vol. 92, no. 1086, pp. 187-193, 2016.

[4] J. Eddy, T. Khan, and F. Schembri, "Medical management of drug-sensitive active thoracic tuberculosis: the work-up, radiographic findings and treatment," Journal of Thoracic Disease, vol. 10, no. S28, pp. S3378-S3391, 2018.

[5] A. J. Crowle, E. J. Ross, and M. H. May, "Inhibition by $1,25(\mathrm{OH})_{2}$-vitamin $\mathrm{D} 3$ of the multiplication of virulent tubercle bacilli in cultured human macrophages," Infection and Immunity, vol. 55, no. 12, pp. 2945-2950, 1987.

[6] A. K. Coussens, A. R. Martineau, and R. J. Wilkinson, "Antiinflammatory and antimicrobial actions of vitamin $\mathrm{D}$ in combating TB/HIV," Scientifica, vol. 2014, Article ID 903680, 13 pages, 2014.

[7] A. R. Martineau, K. A. Wilkinson, S. M. Newton et al., "IFN- $\gamma$ - and TNF-independent vitamin D-inducible human suppression of mycobacteria: the role of cathelicidin LL-37," The Journal of Immunology, vol. 178, no. 11, pp. 7190-7198, 2007.

[8] F. Baeke, T. Takiishi, H. Korf, C. Gysemans, and C. Mathieu, "Vitamin D: modulator of the immune system," Current Opinion in Pharmacology, vol. 10, no. 4, pp. 482-496, 2010.

[9] A. K. Coussens, R. J. Wilkinson, Y. Hanifa et al., "Vitamin $\mathrm{D}$ accelerates resolution of inflammatory responses during tuberculosis treatment," Proceedings of the National Acadamy of Sciences of the United States of America, vol. 109, no. 38, pp. 15449-15454, 2012. 
[10] H.-X. Wu, X.-F. Xiong, M. Zhu, J. Wei, K.-Q. Zhuo, and D.-Y. Cheng, "Effects of vitamin D supplementation on the outcomes of patients with pulmonary tuberculosis: A systematic review and meta-analysis," BMC Pulmonary Medicine, vol. 18, no. 1, article no. 108, 2018.

[11] C. Mathyssen, G. Gayan-Ramirez, R. Bouillon, and W. Janssens, "Vitamin D supplementation in respiratory diseases: Evidence from randomized controlled trials," Polish Archives of Internal Medicine, vol. 127, no. 11, pp. 775-784, 2017.

[12] A. Afzal, R. Rathore, N. F. Butt, and F. A. Randhawa, "Efficacy of vitamin D supplementation in achieving an early sputum conversion in smear positive pulmonary tuberculosis," Pakistan Journal of Medical Sciences, vol. 34, no. 4, pp. 849-854, 2018.

[13] P. Daley, V. Jagannathan, K. R. John et al., "Adjunctive vitamin $\mathrm{D}$ for treatment of active tuberculosis in India: A randomised, double-blind, placebo-controlled trial," The Lancet Infectious Diseases, vol. 15, no. 5, pp. 528-534, 2015.

[14] D. Ganmaa, B. Munkhzul, W. Fawzi et al., "High-dose Vitamin D3 during tuberculosis treatment in Mongolia a randomized controlled trial," American Journal of Respiratory and Critical Care Medicine, vol. 196, no. 5, pp. 628-637, 2017.

[15] A. R. Martineau, P. M. Timms, G. H. Bothamley et al., "Highdose vitamin $\mathrm{D}_{3}$ during intensive-phase antimicrobial treatment of pulmonary tuberculosis: a double-blind randomised controlled trial," The Lancet, vol. 377, no. 9761, pp. 242-250, 2011.

[16] A. Mily, R. S. Rekha, S. M. Kamal et al., "Significant effects of oral phenylbutyrate and vitamin D3 adjunctive therapy in pulmonary tuberculosis: a randomized controlled trial," PLoS ONE, vol. 10, no. 9, Article ID e0138340, 2015.

[17] E. W. Nursyam, Z. Amin, and C. M. Rumende, "The effect of vitamin $\mathrm{D}$ as supplementary treatment in patients with moderately advanced pulmonary tuberculous lesion," Acta Medica Indonesiana, vol. 38, no. 1, pp. 3-5, 2006.

[18] A. P. Ralph, G. Waramori, G. J. Pontororing et al., "L-arginine and vitamin $\mathrm{D}$ adjunctive therapies in pulmonary tuberculosis: a randomised, double-blind, placebo-controlled trial," PLoS ONE, vol. 8, no. 8, Article ID e70032, 2013.

[19] N. Tukvadze, E. Sanikidze, M. Kipiani et al., "High-dose Vitamin D3 in adults with pulmonary tuberculosis: A doubleblind randomized controlled trial," American Journal of Clinical Nutrition, vol. 102, no. 5, pp. 1059-1069, 2015.

[20] R. S. Wallis and A. Zumla, "Vitamin D as adjunctive hostdirected therapy in tuberculosis: A systematic review," Open Forum Infectious Diseases, vol. 3, no. 3, Article ID ofw151, 2016.

[21] J. Wang, M. Feng, S. Ying, J. Zhou, and X. Li, "Efficacy and safety of vitamin D supplementation for pulmonary tuberculosis: A systematic review and meta-analysis," Iranian Journal of Public Health, vol. 47, no. 4, pp. 466-472, 2018.

[22] "Critical appraisal tools. CEBM", https://www.cebm.net/2014/ 06/critical-appraisal/, 2018.

[23] N. Gupta, K. Farooqui, C. Batra, R. Marwaha, and A. Mithal, "Effect of oral versus intramuscular Vitamin D replacement in apparently healthy adults with Vitamin D deficiency," Indian Journal of Endocrinology and Metabolism, vol. 21, no. 1, pp. 131136, 2017. 


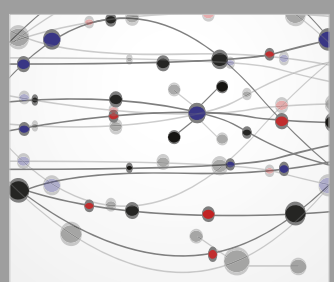

The Scientific World Journal




Gastroenterology Research and Practice

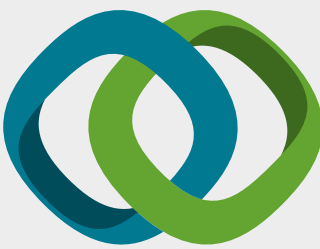

\section{Hindawi}

Submit your manuscripts at

www.hindawi.com


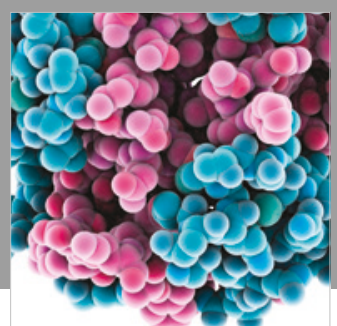

ournal of

Diabetes Research

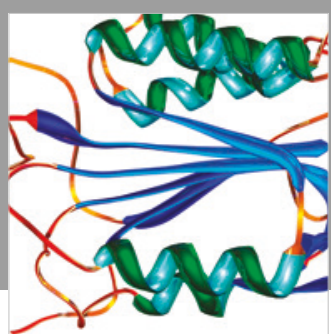

Disease Markers
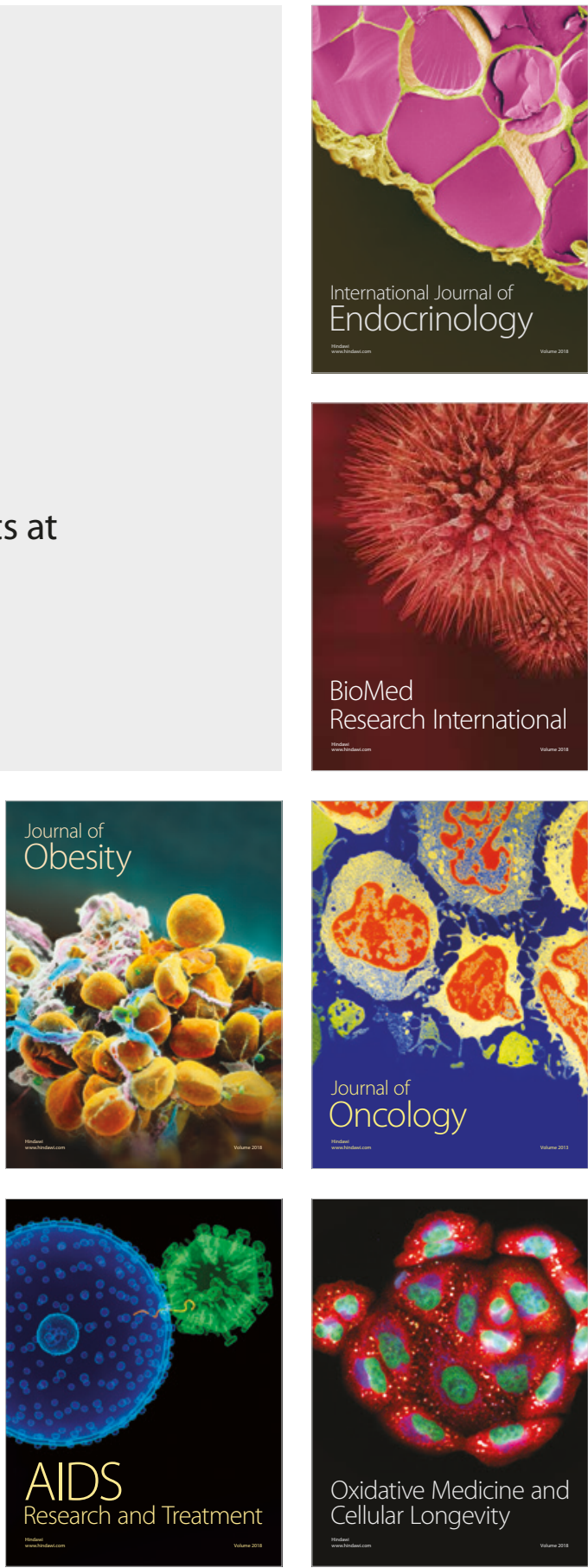